\title{
A FAITHFUL 2-DIMENSIONAL TQFT
}

\author{
STEVAN GAJOVIĆ, ZORAN PETRIĆ AND SONJA TELEBAKOVIĆ ONIĆ
}

\begin{abstract}
It has been shown in this paper that the commutative Frobenius algebra $\mathbb{Q Z}_{5} \otimes Z\left(\mathbb{Q S}_{3}\right)$ provides a complete invariant for two-dimensional cobordisms, i.e., that the corresponding two-dimensional quantum field theory is faithful. Zsigmondy's Theorem is essential to the proof of this result.

Mathematics Subject Classification (2000): 57R56, 18A22, 18D10, 15A69

Keywords: Frobenius algebra, topological quantum field theory, faithful functor, Zsigmondy's Theorem
\end{abstract}

\section{INTRODUCTION}

It is evident that one aspect of topological quantum field theories (TQFTs) deals with the corresponding invariants of manifolds. However, the completeness of these invariants is seldom investigated in the literature. The existence of a faithful $n$ dimensional TQFT provides a complete algebraic invariant for $n$-cobordisms, which may help in their classification.

In the current article we show that there is a faithful 2-dimensional TQFT. As the classification of 2-cobordisms is well-known, this result has a stronger algebraic than topological impact. It shows that there is a commutative Frobenius algebra, which satisfies only the equalities in the language of multiplication, unit, comultiplication and counit, which hold in every commutative Frobenius object.

Since this structure is free of additional equations, one could be tempted to call it "free commutative Frobenius algebra". However, since the category of commutative Frobenius algebras is a groupoid - every homomorphism is an isomorphism (cf. 9, Lemma 2.4.5] - there are no freely generated objects in this category.

Let $\mathcal{F}$ be the category whose objects are symmetric monoidal categories with one distinguished commutative Frobenius object and whose arrows are symmetric monoidal functors preserving distinguished objects and their Frobenius structures. The forgetful functor from $\mathcal{F}$ to the category Set of sets and functions, which maps a symmetric monoidal category to the set of its objects, has a left adjoint, the "free" functor $F$. Let us denote the category $F \emptyset$ by $\mathbf{K}$. This category may be constructed such that its set of objects is the set of finite ordinals and that 1 is the universal commutative Frobenius object, in the same sense as the object 1 of the simplicial category $\Delta$ is the universal monoid. (A detailed construction of $\mathbf{K}$ is given in 3 , Appendix 9.3].)

The standard presentation of the category of 2-cobordisms by generators and relations (cf. [9, Section 1.4]) may be treated as a completeness result (for a logician) or a coherence result (for a category theorist) of the syntax represented by the category $\mathbf{K}$ with respect to the semantics represented by the category of 2-cobordisms. The existence of a commutative Frobenius algebra, which is free of additional equations in the canonical language, provides a new completeness result with the universal object 1 interpreted not as a topological object, but as an algebraic object.

Our result shows that the category of vector spaces contains an isomorphic copy of the category $\mathbf{K}$. Without proving the existence of a faithful 2-dimensional TQFT, 
one cannot be sure that commutative Frobenius objects in the category of vector spaces do not satisfy some additional equations, and, hence, deserve some attributes attached to the standard name "commutative Frobenius algebra". The algebra constructed in this paper justifies this standard name.

There is a result in [5, Section 14] that claims faithfulness of a 1-dimensional TQFT, which is inspired by [4. The third author, in her recent work [15], has shown that every 1-dimensional TQFT, over a field of characteristic zero, is faithful. This means that every such 1-dimensional TQFT provides a complete invariant for 1-cobordisms. On the other hand, we do not know whether there exists a faithful $n$-dimensional TQFT for $n \geq 3$. An important step towards a solution of this problem was given in [8], where the author presents the cobordism category in arbitrary dimension $n$ with generators and relations. Our proof for 2-dimensional case suggests that for $n \geq 3$, particular difficulties could be caused by closed manifolds with many connected components. As it was shown in [6], neither Turaev-Viro, [17, nor Reshetikhin-Turaev, [16, 3-dimensional TQFTs are faithful. We are aware of the fact that even a negative answer to this question might be conclusive - it suggests that TQFTs should find more appropriate targets than the category of vector spaces. Such an approach is provided by extended topological field theories (cf. [10]).

In order to keep this paper as short as possible, we rely on [9] for basic definitions, as well as on now classical works [2, [12], 1], and more recent [10].

\section{The CATEGORY $2 \boldsymbol{C o b}$ AND 2TQFTS}

Let $\mathbf{2} \boldsymbol{C o} \boldsymbol{b}$ be the category whose objects are $\mathbf{0}, \mathbf{1}, \mathbf{2}, \ldots$, where $\mathbf{n}$ is the sequence of $n$ circles and whose arrows are the equivalence classes of 2 -cobordisms defined as in [9, Section 1.2]. We denote cobordisms by $K, L, \ldots$, and $K=L$ means that $K$ and $L$ belong to the same equivalence class. In the illustrations below, cobordisms are oriented top to bottom (the ingoing boundary is at the top and the outgoing boundary is at the bottom of the picture), not left to right as usual.

Let $K: \mathbf{n} \rightarrow \mathbf{m}$ be a 2 -cobordism whose ingoing and outgoing boundaries are respectively the sequences of circles $\left(\Sigma_{0}^{0}, \ldots, \Sigma_{0}^{n-1}\right)$ and $\left(\Sigma_{1}^{0}, \ldots, \Sigma_{1}^{m-1}\right)$. We define an equivalence relation $\rho_{K}$ on the set

$$
(\{0, \ldots, n-1\} \times\{0\}) \cup(\{0, \ldots, m-1\} \times\{1\})
$$

such that $(i, k) \rho_{K}(j, l)$ when $\Sigma_{k}^{i}$ and $\Sigma_{l}^{j}$ belong to the same connected component in $K$ (cf. [3, Section 8]). For example, if $K: \mathbf{3} \rightarrow \mathbf{4}$ is

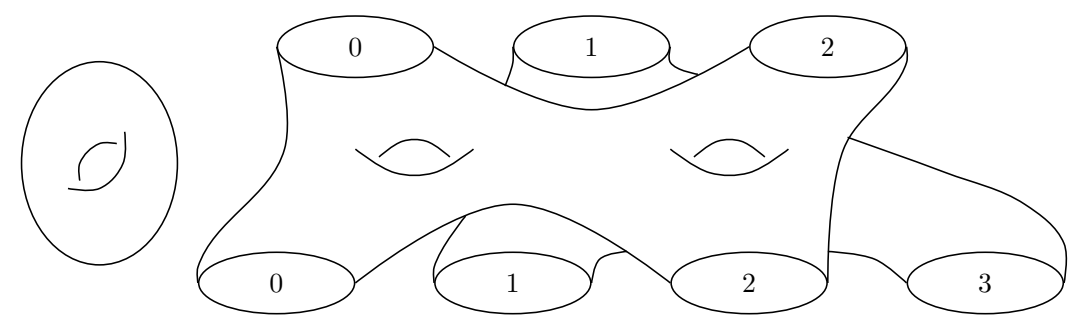

then the equivalence classes of $\rho_{K}$ are

$$
\{(0,0),(2,0),(0,1),(2,1)\} \quad \text { and } \quad\{(1,0),(1,1),(3,1)\} .
$$

Also, we denote by $\left(g_{k}^{i}\right)_{K}$ the genus of the connected component of $K$ containing $\Sigma_{k}^{i}$.

The category $\mathbf{2} \boldsymbol{C o b}$ is a symmetric monoidal with the tensor product $\otimes$ given by "putting side by side" and symmetry generated by the transpositions: 


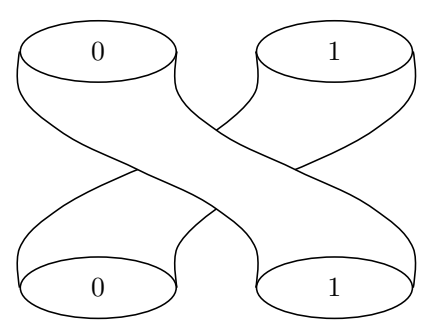

Let $\boldsymbol{V e c t}$ be the category of vector spaces over a fixed field whose symmetric monoidal structure is given by the tensor product and the usual symmetry. According to Atiyah's axioms (see [2, Section 2]), a 2-dimensional quantum field theory (2TQFT) is a symmetric, strong monoidal functor (cf. [11, Section XI.2]) from $2 \mathrm{Cob}$ to Vect.

For $m, k, n \geq 0$, let $E_{m, k, n}$ denote the connected 2-cobordism with $n$ ingoing boundaries, $m$ outgoing boundaries and genus $k$.

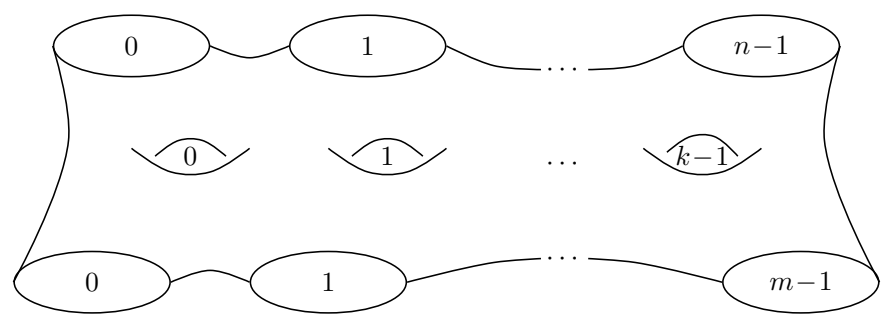

As a part of a relation between 2TQFTs and commutative Frobenius algebras, which is thoroughly explained in [9, Section 3.3], we have that if $F$ is a 2TQFT, then for

$$
\mu=F\left(E_{1,0,2}\right), \quad \eta=F\left(E_{1,0,0}\right), \quad \delta=F\left(E_{2,0,1}\right), \quad \text { and } \quad \varepsilon=F\left(E_{0,0,1}\right),
$$

$(F \mathbf{1}, \mu, \eta, \delta, \varepsilon)$, is a commutative Frobenius algebra. Conversely, if $(A, \mu, \eta, \delta, \varepsilon)$ is a commutative Frobenius algebra, then there is a $2 \mathrm{TQFT}$, which we denote by $F_{A}$, mapping 1 into $A$, and $E_{1,0,2}, E_{1,0,0}, E_{2,0,1}$ and $E_{0,0,1}$ into $\mu, \eta, \delta$ and $\varepsilon$, respectively. For such an $F_{A}$, we denote $F_{A} K$ by $(K)_{A}$, and abbreviate $F_{A} K=F_{A} L$ by $K={ }_{A} L$.

The following three lemmata hold since $2 \mathrm{TQFT}$ is a monoidal functor.

Lemma 2.1 (FILLING HOLES). If $K={ }_{A} L$ for $K, L: \mathbf{n} \rightarrow \mathbf{m}$, then for every $0 \leq i \leq n-1$ and $0 \leq j \leq m-1$, we have

$$
K \circ\left(\operatorname{id}_{i} \otimes E_{1,0,0} \otimes \operatorname{id}_{n-i-1}\right)={ }_{A} L \circ\left(\operatorname{id}_{i} \otimes E_{1,0,0} \otimes \mathrm{id}_{n-i-1}\right)
$$

and

$$
\left(\mathrm{id}_{j} \otimes E_{0,0,1} \otimes \mathrm{id}_{m-j-1}\right) \circ K={ }_{A}\left(\mathrm{id}_{j} \otimes E_{0,0,1} \otimes \mathrm{id}_{m-j-1}\right) \circ L .
$$

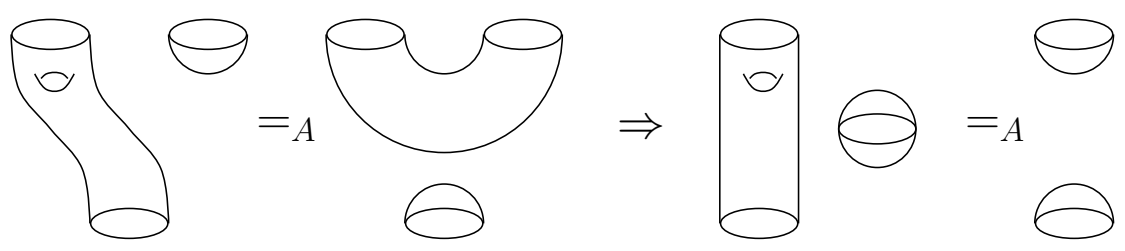

Illustration of the first implication of Lemma 2.1

Lemma 2.2 (STRETCHING 1). If $K={ }_{A} L$ for $K, L: \mathbf{1} \rightarrow \mathbf{0}$, then we have

$$
\left(K \otimes \operatorname{id}_{1}\right) \circ E_{2,0,1}={ }_{A}\left(L \otimes \operatorname{id}_{1}\right) \circ E_{2,0,1},
$$


and if $K={ }_{A} L$ for $K, L: \mathbf{0} \rightarrow \mathbf{1}$, then we have

$$
E_{1,0,2} \circ\left(K \otimes \mathrm{id}_{1}\right)={ }_{A} E_{1,0,2} \circ\left(L \otimes \mathrm{id}_{1}\right) .
$$

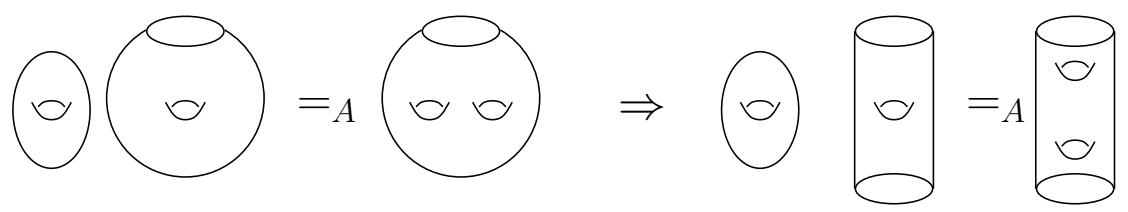

Illustration of the first implication of Lemma 2.2

Lemma 2.3 (STRETCHING 2). If $K={ }_{A} L$ for $K, L: \mathbf{2} \rightarrow \mathbf{0}$, then we have $\left(K \otimes \mathrm{id}_{1}\right) \circ\left(\mathrm{id}_{1} \otimes E_{2,0,0}\right)={ }_{A}\left(L \otimes \mathrm{id}_{1}\right) \circ\left(\mathrm{id}_{1} \otimes E_{2,0,0}\right)$,

and if $K={ }_{A} L$ for $K, L: \mathbf{0} \rightarrow \mathbf{2}$, then we have $\left(\mathrm{id}_{1} \otimes E_{0,0,2}\right) \circ\left(K \otimes \mathrm{id}_{1}\right)={ }_{A}\left(\mathrm{id}_{1} \otimes E_{0,0,2}\right) \circ\left(L \otimes \mathrm{id}_{1}\right)$.
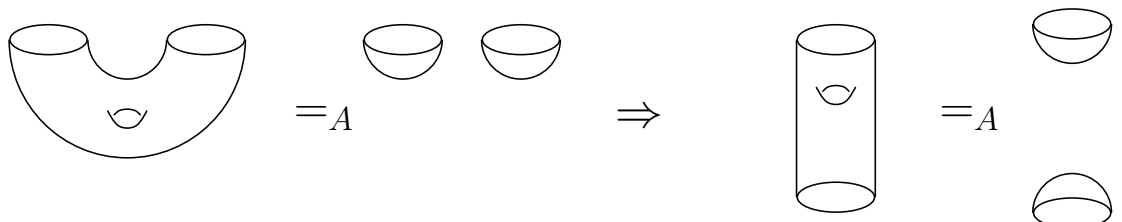

Illustration of the first implication of Lemma 2.3

Proposition 2.4 (MAXIMALITY). If for $K \neq L$, we have $K={ }_{A} L$, where $\operatorname{dim}(A)>1$, then for some $k_{1} \geq \ldots \geq k_{n} \geq 0$ and $l_{1} \geq \ldots \geq l_{m} \geq 0$ such that $\left(k_{1}, \ldots, k_{n}\right) \neq\left(l_{1}, \ldots, l_{m}\right)$, we have

$$
\bigotimes_{i=1}^{n} E_{0, k_{i}, 0}={ }_{A} \bigotimes_{j=1}^{m} E_{0, l_{j}, 0}
$$

Proof. Since $\operatorname{dim}(A)>1$, the cobordisms $K$ and $L$ must have the same source and target. Also, $K \neq L$ entails that either $\rho_{K} \neq \rho_{L}$, or $\rho_{K}=\rho_{L}$ and there is $(i, k)$ such that $\left(g_{k}^{i}\right)_{K} \neq\left(g_{k}^{i}\right)_{L}$, or $\rho_{K}=\rho_{L}$ and for every $(i, k),\left(g_{k}^{i}\right)_{K}=\left(g_{k}^{i}\right)_{L}$ and $K$ and $L$ differ in their closed components.

We start with the last and simplest case. If $\rho_{K}=\rho_{L}$ and for every $(i, k)$ we have $\left(g_{k}^{i}\right)_{K}=\left(g_{k}^{i}\right)_{L}$, then by applying Lemma 2.1 for all the boundary components, we arrive at the equality of the form (2.1).

If $\rho_{K}=\rho_{L}$ and there is $(i, k)$ such that $\left(g_{k}^{i}\right)_{K} \neq\left(g_{k}^{i}\right)_{L}$, then by applying Lemma 2.1 for all the boundary components except the one corresponding to $(i, k)$, and then by applying Lemma 2.2, we arrive at the equality of the form

$$
E_{1, p, 1} \otimes\left(\bigotimes_{i=1}^{n} E_{0, k_{i}, 0}\right)={ }_{A} E_{1, q, 1} \otimes\left(\bigotimes_{j=1}^{m} E_{0, l_{j}, 0}\right)
$$

for some $n, m, p, q \geq 0$ such that $p \neq q$, and $k_{1} \geq \ldots \geq k_{n} \geq 0, l_{1} \geq \ldots \geq l_{m} \geq 0$.

If $\rho_{K} \neq \rho_{L}$ and $(i, k) \rho_{K}(j, l)$, while not $(i, k) \rho_{L}(j, l)$, then by applying Lemma 2.1 for all the boundary components except those corresponding to $(i, k)$ and $(j, l)$ we arrive either directly at the equality of the form

$$
E_{1, p, 1} \otimes\left(\bigotimes_{i=1}^{n} E_{0, k_{i}, 0}\right)={ }_{A} E_{1, q, 0} \otimes E_{0, r, 1} \otimes\left(\bigotimes_{j=1}^{m} E_{0, l_{j}, 0}\right),
$$

for some $n, m, p, q, r \geq 0$ and $k_{1} \geq \ldots \geq k_{n} \geq 0, l_{1} \geq \ldots \geq l_{m} \geq 0$, or this equality is obtained by a further application of Lemma 2.3 .

For $a>\max \left\{k_{1}, l_{1}\right\}$, put the both sides of the equalities (2.2) and (2.3) in the context $E_{0, a, 1}{ }^{\circ} \_\circ E_{1, a, 0}$ in order to obtain the equality of the form (2.1). 


\section{Frobenius algebras $\mathbb{Q Z}_{5}$ and $Z\left(\mathbb{Q S}_{3}\right)$}

For all the examples below, when we fix a basis $\left\langle\beta_{1}, \ldots, \beta_{n}\right\rangle$ of a vector space $V$, then we assume that the tensor product $V \otimes V$ has the fixed basis

$$
\left\langle\beta_{1} \otimes \beta_{1}, \beta_{1} \otimes \beta_{2}, \ldots, \beta_{2} \otimes \beta_{1}, \ldots, \beta_{n} \otimes \beta_{n}\right\rangle,
$$

and we represent the linear transformations by matrices with respect to these bases.

For $\mathbb{Z}_{5}$ being the cyclic group of order 5 , with the generator $a$, let $\mathbb{Q} \mathbb{Z}_{5}$ be the group algebra and let $\left\langle e, a, a^{2}, a^{3}, a^{4}\right\rangle$ be its basis. The multiplication $\mu: \mathbb{Q Z}_{5} \otimes$ $\mathbb{Q Z}_{5} \rightarrow \mathbb{Q Z}_{5}$ is represented by the $5 \times 25$ matrix $M$

$\left[\begin{array}{llllllllllllllllllllllllll}1 & 0 & 0 & 0 & 0 & 0 & 0 & 0 & 0 & 1 & 0 & 0 & 0 & 1 & 0 & 0 & 0 & 1 & 0 & 0 & 0 & 1 & 0 & 0 & 0 \\ 0 & 1 & 0 & 0 & 0 & 1 & 0 & 0 & 0 & 0 & 0 & 0 & 0 & 0 & 1 & 0 & 0 & 0 & 1 & 0 & 0 & 0 & 1 & 0 & 0 \\ 0 & 0 & 1 & 0 & 0 & 0 & 1 & 0 & 0 & 0 & 1 & 0 & 0 & 0 & 0 & 0 & 0 & 0 & 0 & 1 & 0 & 0 & 0 & 1 & 0 \\ 0 & 0 & 0 & 1 & 0 & 0 & 0 & 1 & 0 & 0 & 0 & 1 & 0 & 0 & 0 & 1 & 0 & 0 & 0 & 0 & 0 & 0 & 0 & 0 & 1 \\ 0 & 0 & 0 & 0 & 1 & 0 & 0 & 0 & 1 & 0 & 0 & 0 & 1 & 0 & 0 & 0 & 1 & 0 & 0 & 0 & 1 & 0 & 0 & 0 & 0\end{array}\right]$

while the unit $\eta: \mathbb{Q} \rightarrow \mathbb{Q Z}_{5}$ is represented by the $5 \times 1$ matrix:

$$
\left[\begin{array}{l}
1 \\
0 \\
0 \\
0 \\
0
\end{array}\right]
$$

The comultiplication $\delta: \mathbb{Q Z}_{5} \rightarrow \mathbb{Q Z}_{5} \otimes \mathbb{Q Z}_{5}$ is represented by the $25 \times 5$ matrix $\frac{1}{5} M^{T}$, and the counit $\varepsilon: \mathbb{Q} \mathbb{Z}_{5} \rightarrow \mathbb{Q}$ is represented by the $1 \times 5$ matrix

$$
\left[\begin{array}{lllll}
5 & 0 & 0 & 0 & 0
\end{array}\right] \text {. }
$$

The structure $\left(\mathbb{Q} \mathbb{Z}_{5}, \mu, \eta, \delta, \varepsilon\right)$ is a commutative Frobenius algebra and it is special in the sense that for every $k$

$$
E_{1, k, 1}=\mathbb{Q}_{5} E_{1,0,1} .
$$

Note that $\left(E_{0, k, 0}\right)_{\mathbb{Q} \mathbb{Z}_{5}}$ is represented by the $1 \times 1$ matrix, i.e. the rational number 5 .

Let $Z\left(\mathbb{Q S}_{3}\right)$ be the center of the group algebra $\mathbb{Q S}_{3}$, where $\mathbb{S}_{3}$ is the symmetric group of degree 3 . Denote the three conjugacy classes of $\mathbb{S}_{3}$ by $C_{1}=\{e\}, C_{2}=$ $\{(12),(13),(23)\}$ and $C_{3}=\{(123),(132)\}$. By [7, Proposition 12.22] one can fix

$$
\langle e,(12)+(13)+(23),(123)+(132)\rangle
$$

as the basis of $Z\left(\mathbb{Q S} \mathbb{S}_{3}\right)$.

The multiplication $\mu: Z\left(\mathbb{Q S}_{3}\right) \otimes Z\left(\mathbb{Q S}_{3}\right) \rightarrow Z\left(\mathbb{Q S}_{3}\right)$ is represented by the $3 \times 9$ matrix

$$
\left[\begin{array}{lllllllll}
1 & 0 & 0 & 0 & 3 & 0 & 0 & 0 & 2 \\
0 & 1 & 0 & 1 & 0 & 2 & 0 & 2 & 0 \\
0 & 0 & 1 & 0 & 3 & 0 & 1 & 0 & 1
\end{array}\right],
$$

the unit $\eta: \mathbb{Q} \rightarrow Z\left(\mathbb{Q S}_{3}\right)$ is represented by the $3 \times 1$ matrix

$$
\left[\begin{array}{l}
1 \\
0 \\
0
\end{array}\right]
$$

while the Frobenius form, i.e. the counit $\varepsilon: Z\left(\mathbb{Q S}_{3}\right) \rightarrow \mathbb{Q}$ is represented by the $1 \times 3$ matrix

$$
\left[\begin{array}{lll}
1 & 0 & 0
\end{array}\right] \text {. }
$$

Since the Frobenius pairing $\beta$ is equal to $\varepsilon \circ \mu$ it is represented by the matrix

$$
\left[\begin{array}{lllllllll}
1 & 0 & 0 & 0 & 3 & 0 & 0 & 0 & 2
\end{array}\right]
$$

hence, the corresponding copairing $\gamma$ is represented by the transpose of the matrix

$$
\left[\begin{array}{lllllllll}
1 & 0 & 0 & 0 & \frac{1}{3} & 0 & 0 & 0 & \frac{1}{2}
\end{array}\right] \text {. }
$$


The comultiplication in a Frobenius algebra is given by the composition

$$
(\mathrm{id} \otimes \mu) \circ(\gamma \otimes \mathrm{id})=(\mu \otimes \mathrm{id}) \circ(\mathrm{id} \otimes \gamma),
$$

and the coassociativity of the comultiplication is a consequence of the associativity of the multiplication. Therefore, the comultiplication $\delta: Z\left(\mathbb{Q S}_{3}\right) \rightarrow Z\left(\mathbb{Q S}_{3}\right) \otimes$ $Z\left(\mathbb{Q S}_{3}\right)$ is represented by the $9 \times 3$ matrix, which is the transpose of

$$
\left[\begin{array}{ccccccccc}
1 & 0 & 0 & 0 & \frac{1}{3} & 0 & 0 & 0 & \frac{1}{2} \\
0 & 1 & 0 & 1 & 0 & 1 & 0 & 1 & 0 \\
0 & 0 & 1 & 0 & \frac{2}{3} & 0 & 1 & 0 & \frac{1}{2}
\end{array}\right]
$$

It is easy to check that for the commutative Frobenius algebra $\left(Z\left(\mathbb{Q S}_{3}\right), \mu, \eta, \delta, \varepsilon\right)$ we have that $\left(E_{1,1,1}\right)_{Z\left(\mathbb{Q S}_{3}\right)}$, which is equal to $\mu \circ \delta$, is represented by the matrix

$$
\left[\begin{array}{ccc}
3 & 0 & 3 \\
0 & 6 & 0 \\
\frac{3}{2} & 0 & \frac{9}{2}
\end{array}\right]
$$

Hence, $\left(E_{1, k, 1}\right)_{Z\left(\mathbb{Q S}_{3}\right)}$ is represented by the $k$ th power of this matrix, i.e. by the matrix

$$
\left(\frac{3}{2}\right)^{k-1}\left[\begin{array}{ccc}
2^{2 k-1}+1 & 0 & 2^{2 k}-1 \\
0 & 3 \cdot 2^{2 k-1} & 0 \\
2^{2 k-1}-\frac{1}{2} & 0 & 2^{2 k}+\frac{1}{2}
\end{array}\right]
$$

Eventually, $\left(E_{0, k, 0}\right)_{Z\left(\mathbb{Q S}_{3}\right)}=\varepsilon \circ\left(E_{1, k, 1}\right)_{Z\left(\mathbb{Q S}_{3}\right)} \circ \eta$ is represented by the rational number

$$
\left(\frac{3}{2}\right)^{k-1}\left(2^{2 k-1}+1\right)
$$

\section{Faithfulness}

In this section we denote the tensor product $\mathbb{Q Z}_{5} \otimes Z\left(\mathbb{Q S}_{3}\right)$ by $\mathbb{A}$. The algebra $\mathbb{A}$ is equipped with the commutative Frobenius structure as the tensor product of two such algebras (cf. [9, Section 2.4]). Note that $\left(E_{0, k, 0}\right)_{\mathbb{A}}$ is represented by the rational number

$$
5 \cdot\left(\frac{3}{2}\right)^{k-1}\left(2^{2 k-1}+1\right) .
$$

The following theorem, known as Zsigmondy's Theorem for sums [18] (see also [13. P1.7] and [14]), and the subsequent lemma are crucial for the proof of the faithfulness of the 2 TQFT corresponding to $\mathbb{A}$.

Theorem 4.1. For positive integers $a, b$ and $n$ such that $a, b$ are coprime, $a>b$ and $(n, a, b) \neq(3,2,1)$, there is a prime number $p$ such that $p$ divides $a^{n}+b^{n}$ and for every $k<n, p$ does not divide $a^{k}+b^{k}$.

Lemma 4.2. If for $k_{1} \geq \ldots \geq k_{n} \geq 0$ and $l_{1} \geq \ldots \geq l_{m} \geq 0$

$$
\prod_{i=1}^{n}\left(5 \cdot\left(\frac{3}{2}\right)^{k_{i}-1}\left(2^{2 k_{i}-1}+1\right)\right)=\prod_{j=1}^{m}\left(5 \cdot\left(\frac{3}{2}\right)^{l_{j}-1}\left(2^{2 l_{j}-1}+1\right)\right) \text {, }
$$

then $n=m$ and $\left(k_{1}, \ldots, k_{n}\right)=\left(l_{1}, \ldots, l_{m}\right)$.

Proof. Let $p$ and $q$ be such that $k_{p}, l_{q}>0$ and $k_{p+1}=0=l_{q+1}$ (if there are any). Then the above equality reads

$$
5^{n} \cdot \frac{3^{\left(\sum_{i=1}^{p} k_{i}\right)-p}}{2^{\left(\sum_{i=1}^{p} k_{i}\right)-p}} \prod_{i=1}^{p}\left(2^{2 k_{i}-1}+1\right)=5^{m} \cdot \frac{3^{\left(\sum_{j=1}^{q} l_{j}\right)-q}}{2^{\left(\sum_{j=1}^{q} l_{j}\right)-q}} \prod_{j=1}^{q}\left(2^{2 l_{j}-1}+1\right) .
$$


Since the last digit in $2^{2 k-1}+1$ is either 3 or 9 , such a factor is not divisible by 5 , and we may conclude that $n=m$. Since all the factors but $2^{\left(\sum_{i=1}^{p} k_{i}\right)-p}$ and $2^{\left(\sum_{j=1}^{q} l_{j}\right)-q}$ are odd, we may conclude that $\left(\sum_{i=1}^{p} k_{i}\right)-p=\left(\sum_{j=1}^{q} l_{j}\right)-q$, and that

$$
\prod_{i=1}^{p}\left(2^{2 k_{i}-1}+1\right)=\prod_{j=1}^{q}\left(2^{2 l_{j}-1}+1\right) .
$$

If $\left(k_{1}, \ldots, k_{p}\right) \neq\left(l_{1}, \ldots, l_{q}\right)$, then, after cancelation, we may assume that every $k_{i}$ is different from every $l_{j}$. Assume also that $k_{1}>l_{1}$. It is not possible that $k_{1}=2$, since then $l_{1}=\ldots=l_{q}=1$ and $\left(\sum_{j=1}^{q} l_{j}\right)-q=0<\left(\sum_{i=1}^{p} k_{i}\right)-p$. Hence, $k_{1} \geq 3$ and $2 k_{1}-1 \geq 5$. By applying Zsigmondy's Theorem for sums, there would be a prime that divides $2^{2 k_{1}-1}+1$ and for every $1 \leq j \leq q$ it does not divide $2^{2 l_{j}-1}+1$, which contradicts the above equality.

Theorem 4.3. The $2 \mathrm{TQFT} F_{\mathbb{A}}$ is faithful and injective on objects.

Proof. Since $\operatorname{dim}(\mathbb{A})>1$, the functor $F_{\mathbb{A}}$ is injective on objects. By Lemma 4.2, for every $k_{1} \geq \ldots \geq k_{n} \geq 0$ and $l_{1} \geq \ldots \geq l_{m} \geq 0$ such that $\left(k_{1}, \ldots, k_{n}\right) \neq\left(l_{1}, \ldots, l_{m}\right)$ we have

$$
\bigotimes_{i=1}^{n} E_{0, k_{i}, 0} \neq \bigotimes_{\mathbb{A}} \bigotimes_{j=1}^{m} E_{0, l_{j}, 0},
$$

and it remains for Proposition 2.4 to be applied.

\section{Acknowledgements}

We would like to thank the anonymous referees for suggestions, which helped to improve the paper, and for a comment how to simplify the formulation of Proposition 2.4 and the proof of Theorem 4.3. We also thank Djordje Baralić for some very useful suggestions. This work was supported by projects 174026 and 174032 of the Ministry of Education, Science, and Technological Development of the Republic of Serbia. The first author was supported by DFG-Grant MU 4110/1-1.

\section{REFERENCES}

[1] L. Abrams, Two-dimensional topological quantum field theories and Frobenius algebras, Journal of Knot Theory and its Ramifications, vol. 5 (1996) pp. 569-587

[2] М. Атіуан, Topological quantum field theories, Publications mathématiques de l'IHÉS, vol. 68 (1989) pp. 175-186

[3] Dj. Baralić, Z. Petrić and S. Telebaković, Spheres as Frobenius objects, Theory and Applications of Categories, vol. 33 (2018), pp. 691-726

[4] R. Brauer, On algebras which are connected with semisimple continuous groups, Annals of Mathematics, vol. 38 (1937) pp. 857-872

[5] K. DošEn and Z. Petrić, Symmetric self-adjunctions and matrices, Algebra Colloquium, vol. 19 (Spec 1) (2012) pp. 1051-1082

[6] L. FunAR, Torus bundles not distinguished by TQFT invariants, Geometry and Topology, vol. 17 (2013) pp. 2289-2344

[7] G. JAMES and M. LIEBECK, Representations and Characters of Groups, 2nd Edition, Cambridge University Press, Cambridge, 2001

[8] A. JuHÁsz, Defining and classifying TQFTs via surgery, Quantum Topology, vol. 9 (2018) pp. $229-321$

[9] J. KocK, Frobenius Algebras and 2D Topological Quantum Field Theories, Cambridge University Press, Cambridge, 2003

[10] J. LuRIE, On the Classification of Topological Field Theories, Current Developments in Mathematics, 2008 (D. Jerison et al., editors), International Press, Somerville, MA, 2009, pp. $129-280$

[11] S. MAC LANE, Categories for the Working Mathematician, Springer, Berlin, 1971 (expanded second edition, 1998) 
[12] F. QUinn, Lectures on axiomatic topological quantum field theory, Geometry and Quantum Field Theory (D.S. Freed and K.K. Uhlenbeck, editors), American Mathematical Society, Providence, 1995 , pp. 323-453

13] P. Ribenboim, Catalan's Conjecture, Academic Press, Boston, 1994

[14] M. Roitman, On Zsigmondy primes, Proceedings of the AMS, vol. 125 (1997) pp. 19131919

[15] S. Telebaković, On the Faithfulness of 1-dimensional Topological Quantum Field Theories, preprint, available at ArXiv (2017)

[16] V.G. Turaev, Quantum invariants of knots and 3-manifolds, de Gruyter Studies in Mathematics, vol. 18, Walter de Gruyter \& Co., Berlin, 1994

[17] V.G. Turaev and O.Y. Viro, State sum invariants of 3-manifolds and quantum 6j-symbols, Topology, vol. 31 (1992) pp. 865-902

[18] K. Zsigmondy, Zur Theorie der Potenzreste, Journal Monatshefte für Mathematik, vol. 3 (1892) pp. 265-284

University of Groningen, Bernoulli Institute, Nijenborgh 9, 9747 AG, Groningen, the NetherLANDS

E-mail address: s.gajovic@rug.nl

Mathematical Institute SAnU, Knez Mihailova 36, P.F. 367, 11001 Belgrade, Serbia

E-mail address: zpetric@mi.sanu.ac.rs

University of Belgrade, Faculty of Mathematics, Studentski trg 16, 11000 Belgrade, Serbia

E-mail address: sonjat@matf.bg.ac.rs 